\title{
TRACING POLYCYCLIC AROMATIC HYDROCARBONS AND WARM DUST EMISSION IN THE SEYFERT GALAXY NGC 1068
}

\author{
Justin H. Howell, ${ }^{1}$ Joseph M. Mazzarella, ${ }^{1}$ Ben H. P. Chan, ${ }^{1}$ Steven Lord, ${ }^{2} \mathrm{Jason}_{\text {A. Surace, }}{ }^{3}$ \\ David T. Frayer, ${ }^{2}$ P. N. Appleton, ${ }^{2}$ Lee Armus, ${ }^{3}$ Aaron S. Evans, ${ }^{4}$ Greg Bothun, ${ }^{5}$ \\ Catherine M. Ishida, ${ }^{6}$ Dong-Chan Kim, ${ }^{7}$ Joseph B. Jensen, ${ }^{8}$ Barry F. Madore, ${ }^{1,9}$ \\ David B. Sanders, ${ }^{10}$ Bernhard Schulz, ${ }^{2}$ Tatjana Vavilkin, ${ }^{4}$ \\ Sylvain Veilleux, ${ }^{7}$ and Kevin $\mathrm{Xu}^{2}$ \\ Received 2007 April 10; accepted 2007 July 19
}

\begin{abstract}
We present a study of the nearby Seyfert galaxy NGC 1068 using mid- and far-infrared data acquired with the IRAC, IRS, and MIPS instruments aboard the Spitzer Space Telescope. The images show extensive 8 and $24 \mu \mathrm{m}$ emission coinciding with star formation in the inner spiral approximately $15^{\prime \prime}(1 \mathrm{kpc})$ from the nucleus and a bright complex of star formation $\sim 47^{\prime \prime}(3 \mathrm{kpc}$ ) southwest of the nucleus. The brightest $8 \mu \mathrm{m}$ polycyclic aromatic hydrocarbon (PAH) emission regions coincide remarkably well with knots observed in an $\mathrm{H} \alpha$ image. Strong PAH features at 6.2, 7.7, 8.6, and $11.3 \mu \mathrm{m}$ are detected in IRS spectra measured at numerous locations inside, within, and outside the inner spiral. The IRAC colors and IRS spectra of these regions rule out dust heated by the active galactic nucleus (AGN) as the primary emission source; the spectral energy distributions are dominated by starlight and PAH emission. The equivalent widths and flux ratios of the PAH features in the inner spiral are generally consistent with conditions in a typical spiral galaxy interstellar medium (ISM). Interior to the inner spiral, the influence of the AGN on the ISM is evident via PAH flux ratios indicative of a higher ionization parameter and a significantly smaller mean equivalent width than observed in the inner spiral. The brightest 8 and $24 \mu \mathrm{m}$ emission peaks in the disk of the galaxy, even at distances beyond the inner spiral, are located within the ionization cones traced by $[\mathrm{O} \mathrm{III}] / \mathrm{H} \beta$, and they are also remarkably well aligned with the axis of the radio jets. Although it is possible that radiation from the AGN may directly enhance PAH excitation or trigger the formation of OB stars that subsequently excite PAH emission at these locations in the inner spiral, the orientation of collimated radiation from the AGN and star formation knots in the inner spiral could be coincidental. The brightest PAH- and $24 \mu \mathrm{m}$-emitting regions are also located precisely where two spiral arms of molecular gas emerge from the ends of the inner stellar bar; this is consistent with kinematic models that predict maxima in the accumulation and compression of the ISM, where gas gets trapped within the inner Lindblad resonance of a large stellar bar that contains a smaller, weaker bar.

Key words: galaxies: individual (NGC 1068) — galaxies: Seyfert — infrared: galaxies
\end{abstract}

\section{INTRODUCTION}

Polycyclic aromatic hydrocarbons (PAHs) appear to be ubiquitous in the interstellar medium (ISM) of massive disk galaxies. PAHs emit copiously near regions of active star formation, where molecules are heated by ultraviolet radiation, and are usually as-

\footnotetext{
${ }^{1}$ Infrared Processing and Analysis Center, California Institute of Technology, MS 100-22, Pasadena, CA 91125,USA; jhhowell@ipac.caltech.edu,mazz@ipac .caltech.edu, bchan@ipac.caltech.edu.

2 NASA Herschel Science Center, Infrared Processing and Analysis Center, California Institute of Technology, MS 100-22, Pasadena, CA 91125, USA; lord@ ipac.caltech.edu, frayer@ipac.caltech.edu,apple@ipac.caltech.edu,bschulz@ipac .caltech.edu, cxu@ipac.caltech.edu.

${ }^{3}$ Spitzer Science Center, California Institute of Technology, MS 314-6, Pasadena, CA 91125, USA; jason@ipac.caltech.edu, lee@ipac.caltech.edu.

${ }^{4}$ Department of Physics and Astronomy, State University of New York at Stony Brook, Stony Brook, NY 11794-3800, USA; aevans@orchid.ess.sunysb .edu, vavilkin@grad.physics.sunysb.edu.

${ }^{5}$ Physics Department, University of Oregon, Eugene, OR 97402, USA; nuts@ bigmoo.uoregon.edu.

6 Subaru Telescope, National Astronomical Observatory of Japan, Hilo, HI 96720, USA; cat@subaru.naoj.org.

7 Department of Astronomy, University of Maryland, College Park, MD 20742, USA; kim@astro.umd.edu, veilleux@astro.umd.edu.

${ }^{8}$ Gemini Observatory, 950 North Cherry Avenue, Tucson, AZ 85719, USA; jjensen@gemini.edu.

9 The Observatories of the Carnegie Institution of Washington, 813 Santa Barbara Street, Pasadena, CA 91101, USA; barry@ociw.edu.

${ }^{10}$ Institute for Astronomy, University of Hawaii, 2680 Woodlawn Drive, Honolulu, HI 96822, USA; sanders@ifa.hawaii.edu.
}

sociated with the photodissociation regions (PDRs) in molecular clouds. The infrared spectra of active galaxies generally lack the PAH emission lines characteristic of starburst galaxies (Roche et al. 1991). Freudling et al. (2003) reported the detection of hot dust and weak PAHs in type 1 active galactic nuclei (AGNs), and cooler dust and strong PAHs in type 2 AGNs, similar to the results of Clavel et al. (2000), Buchanan et al. (2006), and P. N. Appleton et al. (2008, in preparation). These results are interpreted as an indication that the high-energy photons from the AGNs destroy PAH molecules exposed to extreme UV radiation from the nucleus, with any observed PAH emission coming from star-forming regions shielded by intervening material (Voit 1992b; Allain et al. 1996; Maloney 1999; Freudling et al. 2003). The Spitzer Space Telescope provides the opportunity to investigate the distribution of PAH emission in a variety of galaxies through its correlation with the zone of influence of the AGN and/or star-forming regions.

NGC 1068 is a prototypical nearby Seyfert 2 galaxy (Antonucci $\&$ Miller 1985) and was the first AGN to be detected (Fath 1909). With an infrared luminosity $L_{\mathrm{ir}}=10^{11.3} L_{\odot}$, NGC 1068 is a luminous infrared galaxy (LIRG) and is a member of the IRAS Revised Bright Galaxy Sample (RBGS; Sanders et al. 2003) consisting of extragalactic objects with $60 \mu \mathrm{m}$ flux greater than $5.24 \mathrm{Jy}$. In addition to the AGN, the galaxy contains a bright inner spiral with active star formation approximately $1 \mathrm{kpc}$ from the nucleus; this structure has often been referred to as a "starburst ring" (Myers \& Scoville 1987; Schinnerer et al. 2000; Spinoglio et al. 2005 ). 

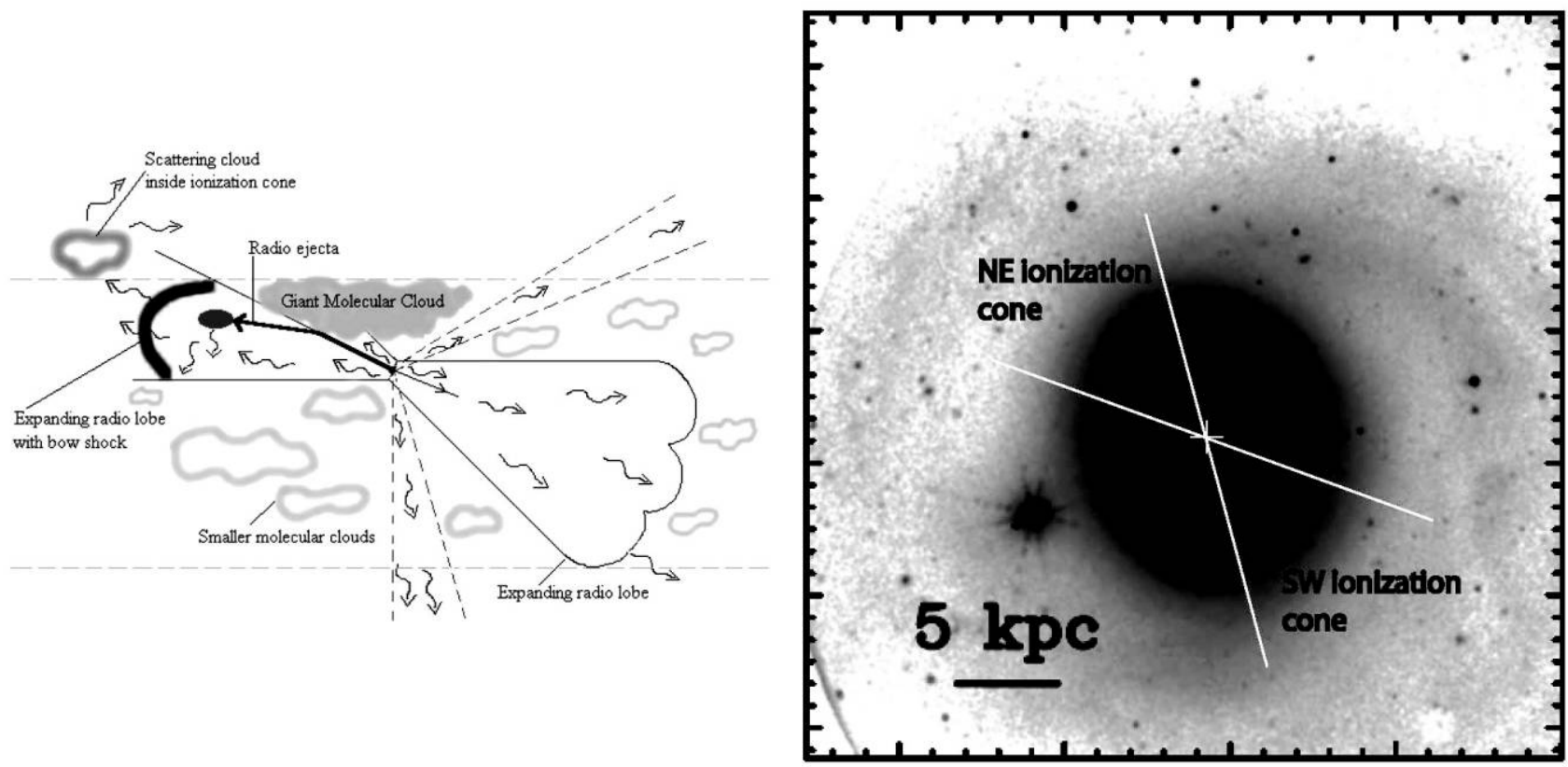

Fig. 1.-Diagram of NGC 1068 ionization cone. Left: View in the plane of the disk, from Bruhweiler et al. (2001). Right: R0 continuum image with ionization cones illustrated (adapted from Veilleux et al. 2003). Large tick marks are at $1^{\prime}$ intervals.

Telesco \& Decher (1988) estimated that star formation would consume the available gas within $0.5 \mathrm{Gyr}$ at current star formation rates. Yuan \& Kuo (1998) showed that the inner spiral resides at the outer Lindblad resonance. The inner spiral arms begin at the ends of a stellar bar (Schinnerer et al. 2000), at the location of peak collisional energy dissipation (Combes \& Gerin 1985). Fabry-Pérot imaging of the $[\mathrm{O}$ III] $\lambda 5007$ line shows clearly defined ionization cones from the center of NGC 1068, inclined such that the disk of the galaxy is illuminated out to $r \sim 11 \mathrm{kpc}$ (Fig. 1, adapted from Bruhweiler et al. 2001 and Veilleux et al. 2003 [Unger et al. 1992; Veilleux et al. 2003]). The [N II] $\lambda \lambda 6548,6583$ line-profile data of Cecil et al. (1990) showed that the kinematics of the ionized gas are well fit by a model in which the ionization cones intersect the plane of the galaxy, rather than illuminating halo gas seen in projection onto the disk. The brightest region of [O III] emission corresponds with the region of peak radio continuum flux, extending $\sim 10^{\prime \prime}$ northeast of the nucleus (Condon et al. 1990; Morganti et al. 1993). A knot of [O III] emission is seen at the distance of the inner spiral, with further [O III] emission extending several kiloparsecs farther out into the disk of the galaxy. Kinematics show that the gas in the ionization cones is ionized in situ by the central engine and is not ejecta from the nuclear region. Spectral line ratios also confirm that the $\mathrm{AGN}$ is the ionizing source even at $r \sim 11 \mathrm{kpc}$ (Veilleux et al. 2003), although the inner spiral itself shows line ratios typical of star-forming regions. The opening angle of the ionization cones is $40^{\circ}$, centered at position angle $31^{\circ}$ (Unger et al. 1992). Veilleux et al. (2003) showed that the [O III] emission is biconical, although it is much fainter to the southwest than the northeast. This is consistent with the conclusion of Cecil et al. (1990) that the ionization cones are inclined with respect to the plane of the disk, illuminating the near side of the disk to the northeast and the far side of the disk to the southwest. Bruhweiler et al. (2001) showed that the $[\mathrm{O}$ III $/ \mathrm{H} \beta$ ratio both interior and exterior to the inner spiral along the cones is consistent with photoionization from the AGN without significant contribution from shocks. Usero et al. (2004) detected the inner spiral in $\mathrm{SiO}$, which is typically interpreted as a signature of shocks. Infrared emission peaks have been detected along the ionization cones at the distance of the inner spiral (Telesco \& Decher 1988; Le Floc'h et al. 2001; Marco \& Brooks 2003). Bland-Hawthorn $\&$ Voit (1993) and Bland-Hawthorn et al. (1997) argued that the IR emission results from dust grains near the surface of molecular clouds heated by the X-ray and UV flux from the AGN. Using the Infrared Space Observatory (ISO), Le Floc'h et al. (2001) detected the peaks in the $7.7 \mu \mathrm{m}$ PAH band, indicating that at least some of the emission is from PAHs rather than large dust grains.

Is it possible that the inner spiral is not illuminated by the AGN, but simply appears to be illuminated due to projection effects? If the inner spiral lies in the plane of the disk, the AGN ionization cones would have to be inclined well out of the plane of the disk and have a small opening angle so as not to intersect it. As noted previously, the $[\mathrm{N} \mathrm{II}]$ and $[\mathrm{O} \mathrm{III}]$ data sets provide strong evidence that the ionization cones intersect the disk. Packham et al. (1997) used near-IR polarimetry to produce a three-dimensional model of the electron scattering cone, concluding that it is inclined by $43^{\circ}$ to the plane of the sky, corresponding to an inclination of $\leq 10^{\circ}$ to the plane of the galaxy. With an opening half-angle of $40^{\circ}$, it clearly intersects the disk. The remaining possibility is that the inner spiral is inclined relative to the disk such that the inner spiral is not illuminated by the AGN. The Packham et al. (1997) model requires an inclination of at least $30^{\circ}$ for the inner spiral to lie outside of the ionization cone. Such an inclination is both physically implausible and would have been easily detected in kinematic data (e.g., $\mathrm{CO}$ or $\mathrm{H} \alpha$ ). We therefore conclude that the AGN illuminates the inner spiral in the region of the PAH knots. The fact that optical-line ratios characteristic of AGN ionization are seen both immediately interior and exterior to the inner spiral indicates that material in the inner spiral cannot be completely shielded from the AGN by intervening material.

In this paper we present the results from analysis of new infrared observations of NGC 1068 from the Spitzer Space Telescope. Observations with the IRAC (Fazio et al. 2004), IRS (Houck et al. 2004), and MIPS (Rieke et al. 2004) instruments are discussed in $\S 2$. Analysis of the data is presented in $\S 3$, and conclusions are given in $\S 4$. 


\section{OBSERVATIONS AND DATA ANALYSIS}

\subsection{Imaging}

NGC 1068 was observed with IRAC by the guaranteed-time observation (GTO) program of Fazio et al. (project ID No. 32). MIPS observations were carried out as part of the Great Observatories All-sky LIRG Survey (GOALS; GO-1 RBGS LIRG/ ULIRG program of Mazzarella et al.; project ID No. 3672). The general data reduction procedures used on the IRAC and MIPS data were described in detail in Mazzarella et al. (2007). Here we discuss the special processing required for NGC 1068 . For reference, the bright star-forming regions are at a radius of $15^{\prime \prime}(1 \mathrm{kpc})$.

Muxbleed saturation artifacts (see the IRAC Data Handbook for details $)^{11}$ in the 5.8 and $8 \mu \mathrm{m}$ images presented challenges in the nuclear region of NGC 1068 within a radius of $15^{\prime \prime}(1 \mathrm{kpc})$. A rough correction for this problem consisted of measuring each artifact in a suitable aperture and subtracting off the flux density of an identical aperture measured at the same galaxy surface brightness level in a region outside the artifact. This procedure resulted in a correction (reduction) of the flux density inside a $15^{\prime \prime}$ aperture by $2.74 \mathrm{Jy}(16 \%)$ at $8.0 \mu \mathrm{m}$ and $0.41 \mathrm{Jy}(5 \%)$ at $5.8 \mu \mathrm{m}$.

Since muxbleed is a result of saturation, an additional correction was needed to account for the lost flux density. Such saturation corrections were estimated by comparison with available data in the literature. The flux density measured within a $6^{\prime \prime}$ diameter aperture was $3.2 \pm 0.2$ Jy at $5.0 \mu \mathrm{m}$ (Rieke \& Low 1972a) and $25 \pm 3 \mathrm{Jy}$ at $10.0 \mu \mathrm{m}$ (Rieke \& Low 1972b). A simple linear interpolation from these values to the IRAC wavelengths predicts $6.2 \pm 0.5$ and $16 \pm 2 \mathrm{Jy}$ at 5.8 and $8.0 \mu \mathrm{m}$, respectively. The blue end of the transmission window of the filter used by Rieke \& Low (1972b) extends to $7.6 \mu \mathrm{m}$ (Low \& Rieke 1971), so the $10 \mu \mathrm{m}$ measurement includes the majority of the flux density from the $7.7 \mu \mathrm{m}$ PAH feature. The Spitzer flux densities measured in a $6^{\prime \prime}$ diameter aperture centered on the nucleus of NGC 1068 are $7.9 \mathrm{Jy}$ at $5.8 \mu \mathrm{m}$ and $7.0 \mathrm{Jy}$ at $8.0 \mu \mathrm{m}$. (We note that the first muxbleed artifact in the $8 \mu \mathrm{m}$ point-spread function [PSF] is located at a radius of about $6^{\prime \prime}$, which is well outside this comparison region with a $6^{\prime \prime}$ diameter.) The $5.8 \mu \mathrm{m}$ value measured from our corrected IRAC data agrees with the estimate based on the Rieke \& Low measurements within the uncertainties of our correction method. However, since our $8 \mu \mathrm{m}$ IRAC estimate is $9 \mathrm{Jy}$ below the estimate based on interpolation of the Rieke \& Low data, we have added $9.0 \mathrm{Jy}$ to the $8 \mu \mathrm{m}$ IRAC aperture measurements for NGC 1068 to account for the flux density lost to saturation.

Photometry of the inner spiral was measured by taking the difference between measurements of $12^{\prime \prime}$ and $18^{\prime \prime}$ radius (800$1200 \mathrm{pc}$ ). Extended-source corrections were applied to each aperture measurement according to the equation derived by T. Jarrett. ${ }^{12}$ The emission peaks on the inner spiral were measured using small elliptical apertures. The wings of the PSF of the bright AGN would bias the observed flux densities if the emission peaks were measured relative to the sky background, so instead the peaks were measured relative to a local background elsewhere on the inner spiral. In the case of the northeast knot, the local background measurements were chosen to also lie along diffraction spikes at 3.6 and $4.5 \mu \mathrm{m}$.

The MIPS $24 \mu \mathrm{m}$ image of NGC 1068 is dominated by a bright unresolved central point source (the AGN) which was saturated within radius $\sim 13^{\prime \prime}$. To measure the spatially resolved features we subtracted a PSF model of the central point source. The IDL pack-

\footnotetext{
11 Available at http://ssc.spitzer.caltech.edu/irac/dh/. .html.
}

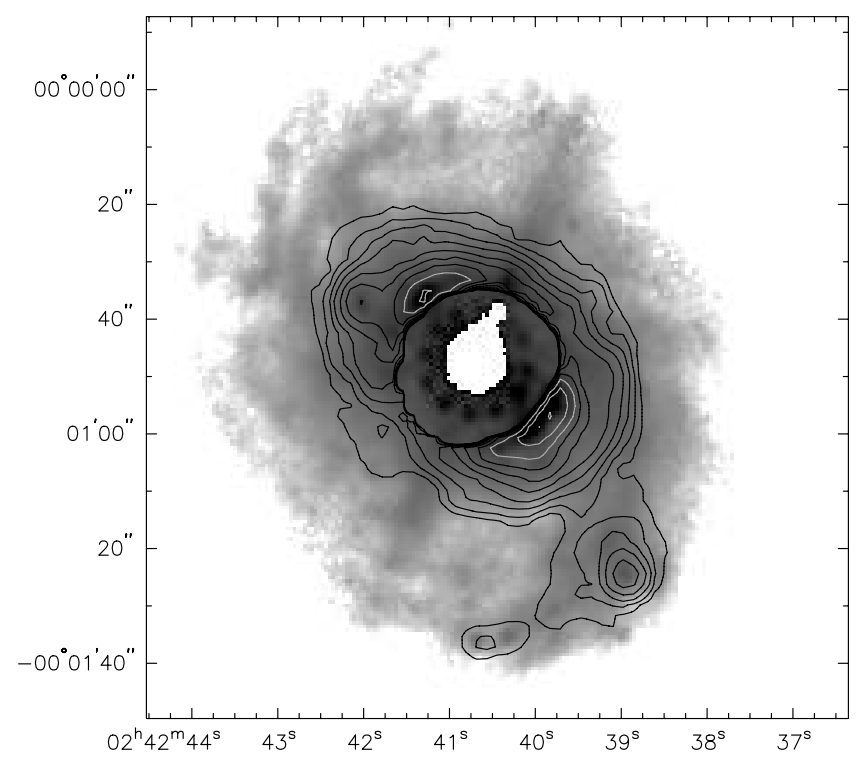

FIG. 2.-The $8 \mu \mathrm{m}$ image of NGC 1068 (gray scale), with contours of the PSF-subtracted $24 \mu \mathrm{m}$ image. The brightest contour levels are shown in light gray for clarity. The saturated data in the nucleus have been excluded from both images. Residual banding is visible on the $8 \mu \mathrm{m}$ image as the streak running southeastnorthwest. The ring of spots is also an artifact of the $8 \mu \mathrm{m}$ PSF. The $24 \mu \mathrm{m}$ data show many of the features seen at $8 \mu \mathrm{m}$, including the two knots on the inner spiral, the northeast tip of the southern arm of the inner spiral $1.5 \mathrm{kpc}\left(23^{\prime \prime}\right)$ east-northeast of the nucleus, and the peak $3 \mathrm{kpc}\left(45^{\prime \prime}\right)$ southwest of the nucleus.

age IDP $3^{13}$ was used to centroid the galaxy and PSF images; then, the scale factor of the PSF image was interactively adjusted to best subtract off the point-source component of the galaxy image. As recommended by the MIPS Data Handbook, ${ }^{14}$ an object of similar color was chosen to create the PSF image. The ULIRG IRAS F05189-2524 served as a satisfactory PSF for this purpose, as it is both very bright and unresolved by Spitzer at $24 \mu \mathrm{m}$. IRAS F05189-2524 is also a Seyfert 2 galaxy, and it is the best match to the IRAS $60 \mu \mathrm{m} / 25 \mu \mathrm{m}$ color of NGC 1068, with a color of 3.82 , compared to 2.24 for the latter. The only galaxies in the GOALS sample with colors more closely matching NGC 1068 were spatially resolved in the MIPS $24 \mu \mathrm{m}$ images. Contours of the PSF-subtracted $24 \mu \mathrm{m}$ image of NGC 1068 are overlaid on the $8 \mu \mathrm{m}$ image in Figure 2. The elongation of the masked region on the $8 \mu \mathrm{m}$ image is due to the muxbleed residuals discussed above.

The best-fit PSF for NGC 1068 was also used to estimate the total $24 \mu \mathrm{m}$ flux density, which could not be measured directly due to saturation. The point-source component of the total flux density was estimated by taking the flux density of IRAS F051892524 (3.04 Jy) and multiplying by the best-fitting PSF scale factor (25), resulting in an estimate of $75.90 \mathrm{Jy}$. In addition, the extended $24 \mu \mathrm{m}$ emission from NGC 1068 was measured directly from the PSF-subtracted image. Combining the $0.5 \mathrm{Jy}$ extended component with the point-source component yields an estimated total $24 \mu \mathrm{m}$ flux density of NGC 1068 (before aperture and color corrections) of $76.4 \mathrm{Jy}$. The uncertainty in the empirically determined PSF scale factor (25.0) is about $20 \%$, and this dominates the uncertainty in this $24 \mu \mathrm{m}$ total flux density measurement.

Table 1 presents the Spitzer flux density measurements and estimated uncertainties. As described in detail in Mazzarella et al. (2007) MIPS color corrections have been applied based on the IRAS and MIPS spectral energy distributions (SEDs). With aperture and color corrections, the total estimated $24 \mu \mathrm{m}$ flux density

\footnotetext{
13 Available at http://mips.as.arizona.edu/mipspage/IDP3/idp3example.html.

14 Available at http://ssc.spitzer.caltech.edu/mips/dh/.
} 
TABLE 1

Spitzer Flux Density Measurements

\begin{tabular}{|c|c|c|c|c|c|c|c|c|}
\hline \multirow[b]{2}{*}{ FEATURE WITHIN NGC 1068} & \multicolumn{5}{|c|}{ IRAC } & \multicolumn{3}{|c|}{ MIPS } \\
\hline & $\begin{array}{l}\text { Aperture Radius } \\
\quad(\operatorname{arcsec})\end{array}$ & $\begin{array}{l}\text { Aperture Radius } \\
\qquad(\mathrm{kpc})\end{array}$ & $\begin{array}{c}3.6 \mu \mathrm{m} \\
\text { (Jy) }\end{array}$ & $\begin{array}{l}4.5 \mu \mathrm{m} \\
(\mathrm{Jy})\end{array}$ & $\begin{array}{c}5.8 \mu \mathrm{m} \\
\text { (Jy) }\end{array}$ & $\begin{array}{c}8 \mu \mathrm{m} \\
(\mathrm{Jy})\end{array}$ & $\begin{array}{c}24 \mu \mathrm{m} \\
\text { (Jy) }\end{array}$ & $\begin{array}{c}70 \mu \mathrm{m} \\
\text { (Jy) }\end{array}$ \\
\hline Total & $90^{\prime \prime}$ & 6 & 3.8 & 5.1 & 13 & 23 & 80 & 180 \\
\hline $1 \mathrm{kpc}$ & $15^{\prime \prime}$ & $\ldots$ & 2.8 & 4.3 & 9.0 & 17 & $\ldots$ & $\ldots$ \\
\hline Northeast PAH knot..................... & $4^{\prime \prime}$ & $0.26^{\mathrm{a}}$ & 0.011 & 0.006 & 0.05 & 0.11 & $\ldots$ & $\ldots$ \\
\hline Southwest PAH knot................... & $5^{\prime \prime}$ & $0.33^{\mathrm{a}}$ & 0.024 & 0.020 & 0.11 & 0.26 & $\ldots$ & $\ldots$ \\
\hline 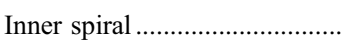 & $12^{\prime \prime}-18^{\prime \prime}$ & $0.8-1.2$ & 0.29 & 0.20 & 0.80 & 1.85 & $\ldots$ & $\ldots$ \\
\hline
\end{tabular}

NotEs.- The approximate uncertainties are indicated by the number of significant figures; specifically, $10 \%$ at IRAC wavelengths and $20 \%$ at $70 \mu \mathrm{m}$. See text for a discussion of the $24 \mu \mathrm{m}$ uncertainties.

${ }^{a}$ Effective radius of custom aperture. Flux densities for the PAH knots are relative to the local background elsewhere in the inner spiral.

using the Spitzer data is 79.6 Jy. Comparison with the IRAS $25 \mu \mathrm{m}$ measurements of $87.6 \pm 0.2$ Jy (Sanders et al. 2003) indicates that this $24 \mu \mathrm{m}$ estimate from Spitzer is low by about $9 \%$. This is consistent with the systematic mean offset of $20 \%$ (with a $1 \sigma$ scatter of 15\%) observed between the $24 \mu \mathrm{m}$ Spitzer measurements and $25 \mu \mathrm{m} I R A S$ measurements across our entire sample of 203 systems (Mazzarella et al. 2007). We are therefore confident in the PSF-fitting procedure, from which it can be stated that more than $99 \%(75.9 / 76.4)$ of the $24 \mu \mathrm{m}$ emission from NGC 1068 originates within a radius of $\approx 30^{\prime \prime}$ (the first Airy ring). Approximately $80 \%$ of the $24 \mu \mathrm{m}$ emission originates within the saturated region $r<13^{\prime \prime}$. NGC 1068 also saturated the $160 \mu \mathrm{m}$ array, and a flux density estimate could not be recovered.

\subsection{Spectroscopy}

The nucleus of the galaxy was observed at high spectral resolution by the GTO program of Houck et al. (program ID No. 14), and mapped at low resolution by the GO-1 program of Gallimore et al. (program ID No. 3269). The Short-Low (SL) IRS maps consisted of 13 half slit-width $\left(1.8^{\prime \prime}\right)$ steps for the first- and secondorder slits. The maps were centered on the nucleus. There were no dedicated off (sky) observations, but a local background was subtracted by using the nonprimary slit, which is centered approximately $80^{\prime \prime}$ from the nucleus, for both the SL1 and SL2 maps. The IRAC $8 \mu \mathrm{m}$ surface brightness at the locations selected for the background are typically $10^{-4}$ to $10^{-3}$ of the level at the nucleus. CUBISM $^{15}$ was used to perform the local background subtraction and form the SL spectral cubes. From these cubes we were able to identify regions over which to extract one-dimensional spectra, and we used CUBISM to perform this extraction in matched apertures in SL1 and SL2. In addition, we used CUBISM to generate a pure PAH map over the area covered by the IRS slits. Aperture locations and the PAH map are shown in Figure 3. Fluxes and equivalent widths of the low-resolution data were measured using PAHFIT (Smith et al. 2007). The regions closest to the nucleus suffered from saturation and did not yield usable spectra; see Figure 3 (bottom). Table 2 presents the equivalent widths and line fluxes of the emission features measured in each aperture.

The Short-High and Long-High IRS observations do not suffer from saturation, but they only cover a single position at the center of the galaxy. No background subtraction was performed due to the lack of a background-slit observation. Spectra were extracted using SPICE, ${ }^{16}$ and lines were measured on the extracted

\footnotetext{
${ }_{15}^{15}$ Available at http://turtle.as.arizona.edu/jdsmith/cubism.php.

${ }^{16}$ Available at http://ssc.spitzer.caltech.edu/postbcd/spice.html.
}

spectra using SMART (Higdon et al. 2004). Our high-resolution spectral line measurements are presented in Table 3.

\subsection{Separating Nonstellar from Stellar Emission in Seyfert Galaxies}

Although IRS spectra provide the ideal measure of the presence and strength of PAH emission, the available data cover only a portion of the inner spiral. IRAC and MIPS imaging is required to investigate the region outside of the spectral map. There are several possible sources for the IR emission from Seyfert galaxies. The power-law continuum from an AGN can contribute to all IRAC wavelengths. The 3.6 and $4.5 \mu \mathrm{m}$ data are dominated by the Rayleigh-Jeans tail of stellar photospheric emission. The $5.8 \mu \mathrm{m}$ channel covers the wavelength range that includes the $6.2 \mu \mathrm{m}$ PAH line, while the $8 \mu \mathrm{m}$ channel includes the 7.7 and 8.6 $\mu \mathrm{m}$ PAH emission features. Hot dust can also be a significant contribution at all IRAC wavelengths. Stellar continuum emission is insignificant compared to PAH emission in the IRAC $8 \mu \mathrm{m}$ band in nearly all nearby LIRGs (Mazzarella et al. 2007). The stellar continuum flux density at $8 \mu \mathrm{m}$ is estimated to be $19 \%-23 \%$ of the $3.6 \mu \mathrm{m}$ flux density (Pahre et al. 2004; Dale et al. 2005); based on this we estimate that stars contribute only a few percent (for example, NGC $10681 \mathrm{kpc}$ aperture: $2.8 \times$ $0.23 / 17=0.038$ ) of the $8 \mu \mathrm{m}$ flux densities of this galaxy. To test for spatial variations between the starlight and PAH emission in NGC 1068, a scaled $3.6 \mu \mathrm{m}$ image was subtracted from the $8 \mu \mathrm{m}$ image. The ratio of this nonstellar emission image to the $8 \mu \mathrm{m}$ image is constant with little variation across the galaxy (excluding the central region, which saturated at $8 \mu \mathrm{m}$, as noted previously), showing that starlight accounts for $\sim 4 \%$ of the $8 \mu \mathrm{m}$ emission throughout the disk of NGC 1068.

Determining the presence of PAHs using IRAC and MIPS photometry requires the measurement and modeling of the contribution of AGN continuum and hot dust at $8 \mu \mathrm{m}$. Several color-color diagnostics have been used to distinguish AGN-dominated systems and starburst-dominated systems in the literature (e.g., Stern et al. 2005). The GOALS sample (Mazzarella et al. 2007) is shown in the [3.6]- [4.5] versus [4.5]-[8] color-color space in Figure 4. Also shown are the colors of hot dust and AGN power laws. Since the vertical axis is the ratio of two channels normally dominated by starlight, galaxies without significant AGNs or hot dust lie near zero. The horizontal axis separates galaxies with little or no PAH emission (left) from those dominated by PAH emission (right). The [4.5]-[8] color measures the jump from the Rayleigh-Jeans tail of starlight at $4.5 \mu \mathrm{m}$ (plus AGN continuum, if present) to the bright PAH emission at $8 \mu \mathrm{m}$. The better known Stern et al. 

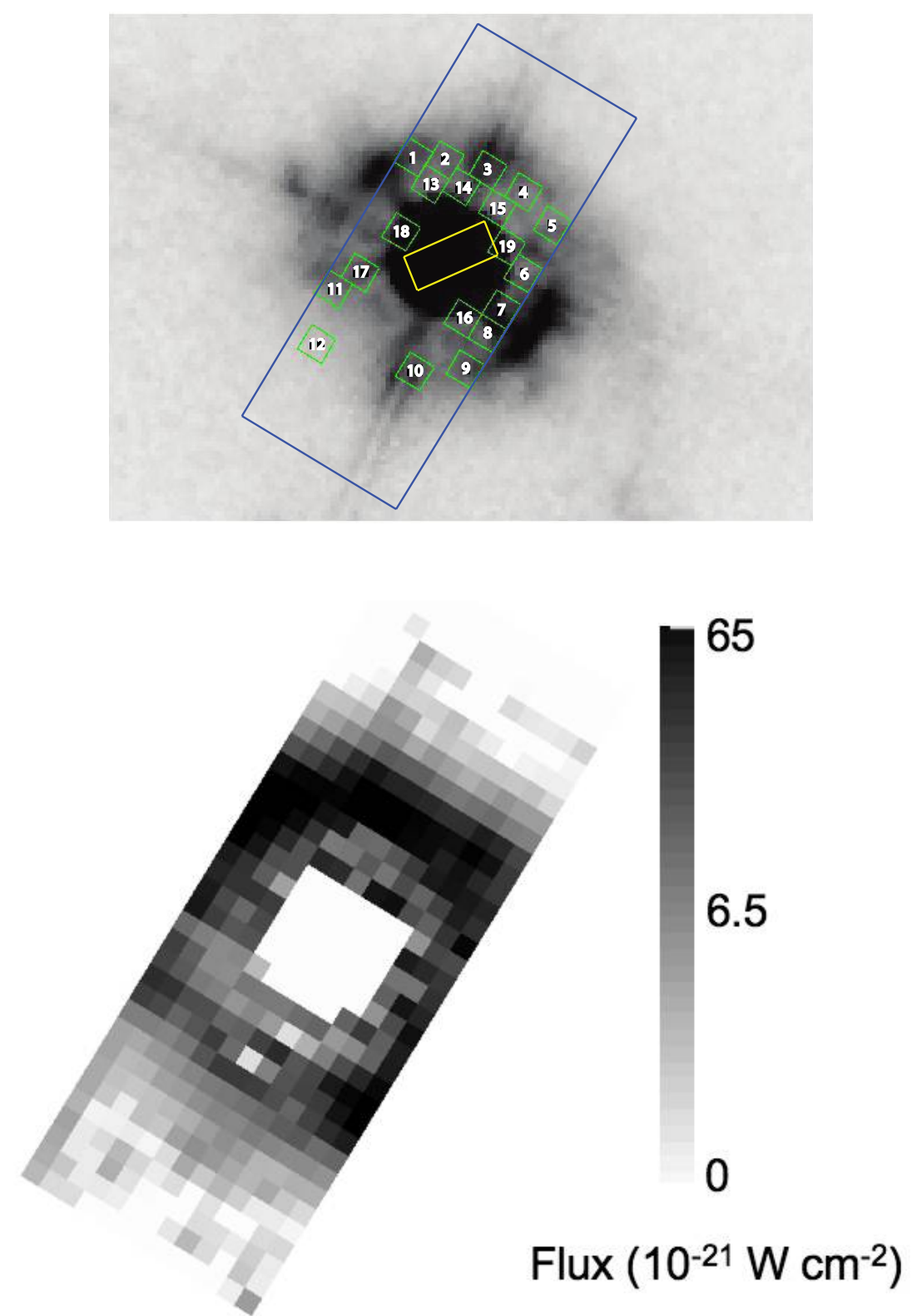

FIG. 3.-Extraction apertures for the SL IRS spectra are overlaid on the $5.8 \mu \mathrm{m}$ IRAC mosaic (top, green numbered boxes). Also shown are the area covered by the SL map (blue box) and the position of the SH slit ( yellow box). Banding and PSF artifacts are as described in Fig. 2. North is up, and east is to the left. The map of the 6.2 $\mu \mathrm{m}$ $\mathrm{PAH}$ feature is shown in the bottom panel. The center of the galaxy has been masked out.

color-color space uses [5.8]-[8] on the horizontal axis instead of [4.5]-[8]. Since both the 5.8 and $8 \mu \mathrm{m}$ channels include PAH bands, [4.5]-[8] is preferred due to the clearer physical interpretation.

Combinations of IRAC images can be used to create spatial maps of different components of IR emission. Pahre et al. (2004) used 3.6 and $4.5 \mu \mathrm{m}$ data to estimate the contribution of starlight, which was then scaled down in proportion to a Rayleigh-Jeans tail and subtracted from the $8 \mu \mathrm{m}$ image to create a map of nonstellar (typically PAH) emission. As noted above, this technique has little effect on LIRG and ULIRG data such as those examined here since the $8 \mu \mathrm{m}$ images are dominated by nonstellar emission. A similar method can be used to identify nonstellar emission at $4.5 \mu \mathrm{m}$. No PAH emission lines are present at such short wavelengths, so the nonstellar emission sources are direct radiation from the AGN and hot dust only. Making an assumption about the form of the nonstellar emission, this technique can be used to quantitatively estimate the emission from stars and the assumed nonstellar source at 3.6 and $4.5 \mu \mathrm{m}$. The ratio of stellar emission at $3.6 \mu \mathrm{m}$ to that at $4.5 \mu \mathrm{m}, f_{*}$, is known; using Pahre et al. (2004), $f_{*}=1.7$. A similar ratio, $f_{\mathrm{pl}}$, is easily calculated for any given power-law slope $\alpha$. For typical values $0.84<\alpha<1.4$, $0.83<f_{\mathrm{pl}}<0.73$. Image subtraction is then performed, with the power-law component of the $4.5 \mu \mathrm{m}$ image given by $I_{\mathrm{pl}}(4.5)=$ $\left[f_{*} I(4.5)-I(3.6)\right] /\left(f_{*}-f_{\mathrm{pl}}\right)$, where $I(\lambda)$ is the IRAC image at a given wavelength. Similar equations are easily derived for the stellar components of each image or the power-law component of the $3.6 \mu \mathrm{m}$ image. Note that if the nonstellar emission arises from hot dust this method will only reveal the locations of such emission, and one can simply use $I(4.5)-I(3.6) / f_{*}$ without attempting to account for the nonstellar contribution to the $3.6 \mu \mathrm{m}$ flux (Engelbracht et al. 2005). A quantitative flux density estimate 


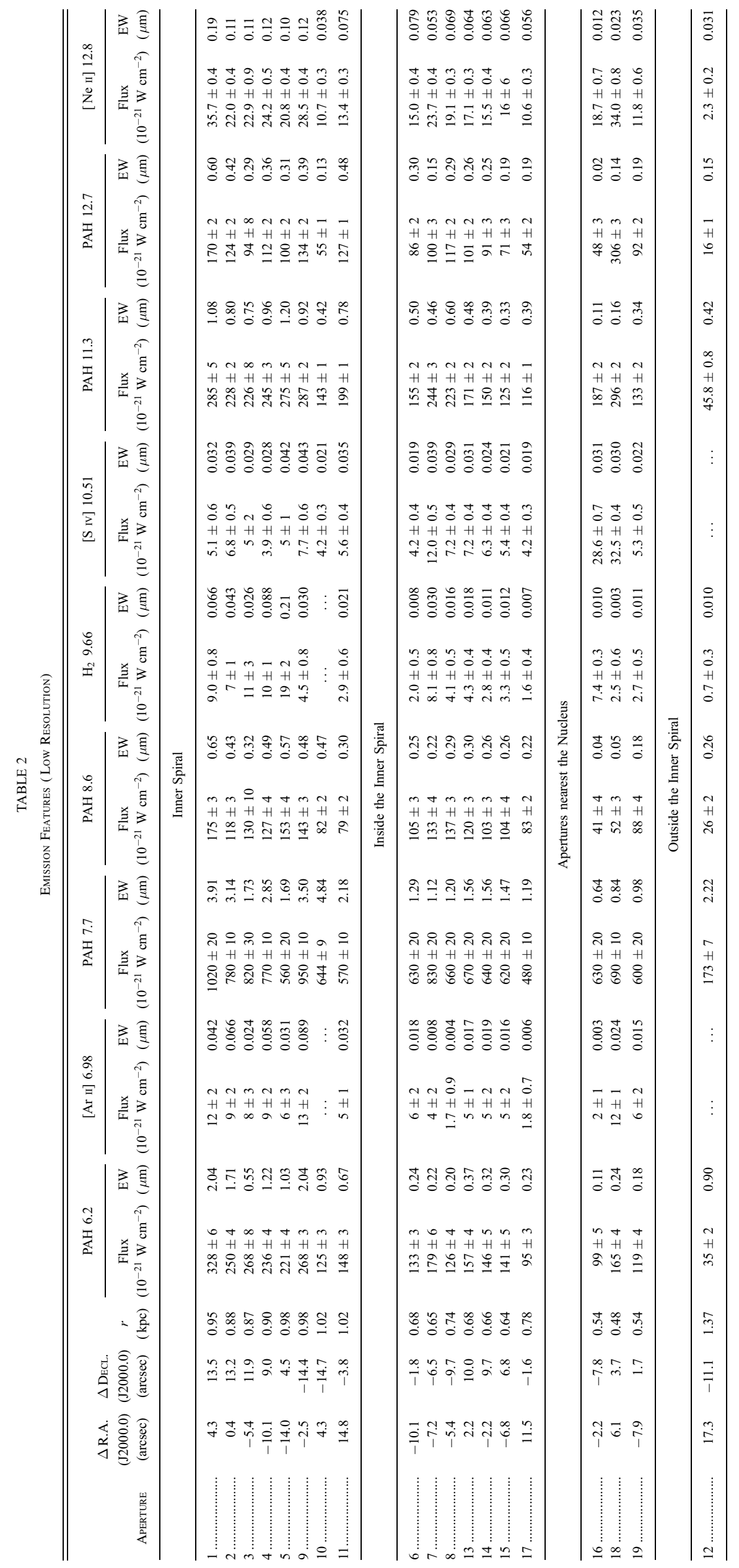


TABLE 3

Nuclear Emission Features (High Resolution)

\begin{tabular}{|c|c|c|}
\hline Line & $\begin{array}{c}\text { Flux } \\
\left(10^{-21} \mathrm{~W} \mathrm{~cm}^{-2}\right)\end{array}$ & $\begin{array}{l}\text { Equivalent Width } \\
\qquad(\mu \mathrm{m})\end{array}$ \\
\hline 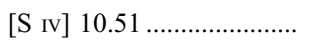 & $460 \pm 60$ & 0.006 \\
\hline$[\mathrm{Ne}$ II $] 12.81 \ldots \ldots \ldots \ldots \ldots \ldots$ & $520 \pm 60$ & 0.008 \\
\hline$[\mathrm{Ne} \mathrm{v}] 14.3 \ldots \ldots \ldots \ldots \ldots \ldots$ & $1110 \pm 60$ & 0.017 \\
\hline 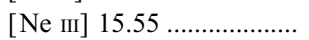 & $2100 \pm 200$ & 0.024 \\
\hline [S III] $18.71 \ldots \ldots \ldots \ldots \ldots \ldots$ & $220 \pm 20$ & 0.004 \\
\hline$[\mathrm{Ne} v] 24.31 \ldots \ldots \ldots \ldots \ldots \ldots \ldots$ & $800 \pm 100$ & 0.023 \\
\hline 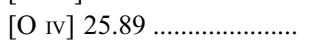 & $2200 \pm 200$ & 0.077 \\
\hline [S II] $33.48 \ldots \ldots \ldots \ldots \ldots \ldots \ldots$ & $420 \pm 60$ & 0.025 \\
\hline 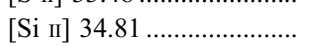 & $620 \pm 20$ & 0.034 \\
\hline$[\mathrm{Ne}$ II] $36.0 \ldots \ldots \ldots \ldots \ldots \ldots \ldots$ & $130 \pm 20$ & 0.006 \\
\hline
\end{tabular}

would require an assumed dust temperature from which $f_{\text {dust }}$ could be calculated, replacing $f_{\mathrm{pl}}$ in the above prescription. In either case, the assumed nonstellar emission can also be extended to $8 \mu \mathrm{m}$ to better constrain the PAH emission.

\section{DISCUSSION}

\subsection{Spitzer Data}

The IRAC colors of the region within $1 \mathrm{kpc}$ of the nucleus and the total emission from the galaxy place NGC 1068 in the AGN region of the IRAC color-color diagram defined by Stern et al. (2005). Emission at IRAC wavelengths is highly concentrated, with $70 \%-80 \%$ of the flux density coming from the central kiloparsec (Table 1).

High-resolution spectra of the nucleus show strong AGN signatures in a number of diagnostic line ratios. Line fluxes and equivalent widths are presented in Table 3 . The [O Iv] $25.9 \mu \mathrm{m} /$ [Ne II] $12.8 \mu \mathrm{m}$ line ratio is used as a diagnostic for the fractional contribution of the AGN to the luminosity of a galaxy (Genzel et al. 1998; Armus et al. 2007). The NGC 1068 nucleus has a ratio of 4.2 as measured from the IRS spectra, compared to 2.7 as measured from the $I S O$ data of Sturm et al. (2002). [Ne v] $14.3 \mu \mathrm{m} /$ [Ne II] $12.8 \mu \mathrm{m}$ is a similar diagnostic ratio; the nucleus of NGC 1068 has a ratio of 2.1 , compared to the $I S O$ ratio of 1.4. Although these AGN diagnostic ratios are higher than the ISO measurements of Sturm et al. (2002) this is not surprising since the IRS slits are smaller than the corresponding $I S O$ aperture by roughly a factor of 2. With less light from the disk of the galaxy, the line ratios might be expected to show higher ionization. Using the [Ne v] $14.3 \mu \mathrm{m} /[\mathrm{Ne}$ III] $15.6 \mu \mathrm{m}$ and [Ne II] $12.8 \mu \mathrm{m} /[\mathrm{Ne}$ III] $15.6 \mu \mathrm{m}$ line ratios measured using the high-resolution spectrum, the diagnostic diagram of Voit (1992a) indicates an ionization parameter $\log U \sim-2$ and a power-law input spectrum of index $\alpha \sim-1.3$. Compared to nearby AGNs, starbursts, and ULIRGs (Armus et al. 2007), the line ratios in the nucleus of NGC 1068 are in excellent agreement with the AGN sample, consistent with a $100 \%$ AGN contribution to the observed mid-IR nuclear spectrum.

The IRAC images show bright $8 \mu \mathrm{m}$ peaks (also detected in the other IRAC channels) along the northeast and southwest arcs at P.A. $\sim 45^{\circ}$ in the inner spiral (Fig. 2) at the locations first detected by Telesco \& Decher (1988). Of the total $8 \mu \mathrm{m}$ flux density within the annulus $800 \mathrm{pc}<R<1200 \mathrm{pc}$ from the nucleus, $52 \%$ is emitted by these two knots. The surface brightness of the knots (400-500 $\left.\mathrm{MJy} \mathrm{sr}^{-1}\right)$ is more than twice that seen elsewhere in the inner spiral $\left(\sim 200 \mathrm{MJy} \mathrm{sr}^{-1}\right)$. Compared to the central kiloparsec of the galaxy, these knots are bluer by more than half a magnitude in [3.6] - [4.5] and redder by $0.55-0.85 \mathrm{mag}$ in [4.5][8]. Since the wavelength range of the $8 \mu \mathrm{m}$ channel includes both

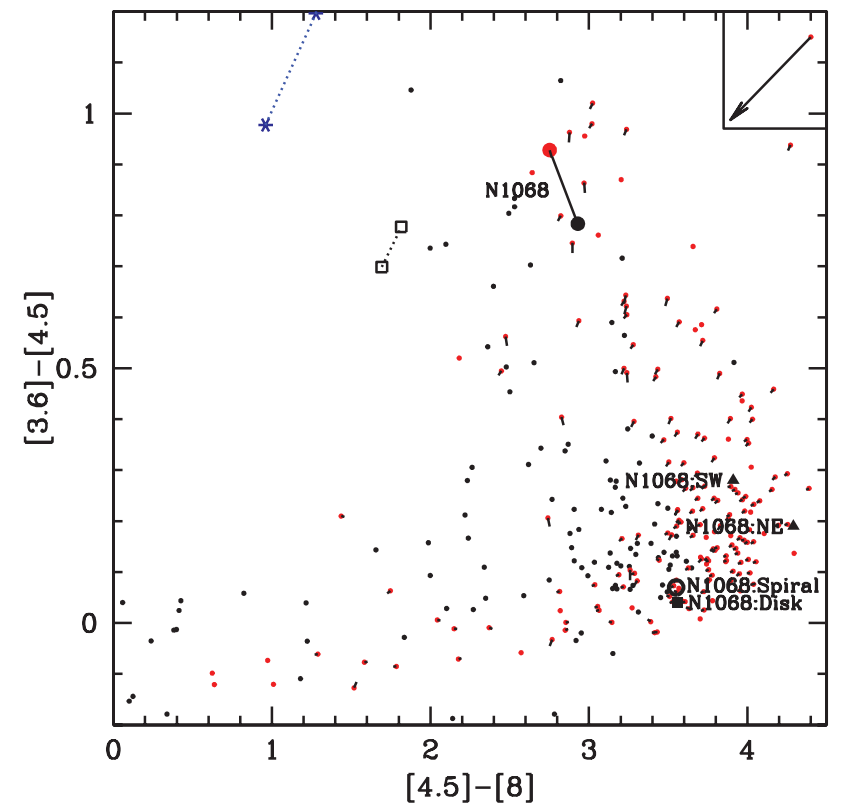

FIG. 4.-IRAC colors of the RBGS LIRGs and ULIRGs from Mazzarella et al. (2007). NGC 1068 is marked (1 kpc radius [red circles] and total apertures [black circles]), as are the colors of the two bright knots on the inner spiral and the colors of the galaxy disk $(r>1 \mathrm{kpc})$ and the inner spiral. Small red circles indicate the central $1 \mathrm{kpc}$ radius aperture measurements of the rest of the GOALS sample, while black circles indicate total magnitudes. Unit vectors indicating the change in color from the central aperture to the total aperture are shown; note that there is not a one-to-one correspondence between central and total aperture measurements due to a combination of resolution and multiple interacting galaxies. The median change in color from the central $1 \mathrm{kpc}$ aperture to the total aperture is shown by the arrow at the top right. Colors are expressed in the Vega magnitude system, so by definition a pure starlight SED would appear near zero in both color axes. Also shown are the colors of a pure AGN power-law spectrum (open squares; from lower left to upper right: $\alpha=0.84$, Clavel et al. 2000; $\alpha=1.4$, Neugebauer et al. 1987), and hot dust (blue circles; for 1000 and $900 \mathrm{~K}$ ).

the 7.7 and $8.6 \mu \mathrm{m}$ PAH features, the latter color strongly suggests that the knots are dominated by PAH emission.

The low-resolution IRS data confirm the predominance of PAH emission in the knots observed in the IRAC $8 \mu \mathrm{m}$ image. The 6.2, 7.7, and $11.2 \mu \mathrm{m}$ PAH features, plus the PAH $12.7 \mu \mathrm{m}+$ [Ne II] $12.8 \mu \mathrm{m}$ blend, are clearly visible throughout the inner spiral. Figure 5 shows representative spectra, including the inner spiral, the region interior to the inner spiral, and the region outside of the inner spiral. Fluxes and equivalent widths of each measured emission feature are presented in Table 2. The PAH features are strongest in the inner spiral (apertures 1-5, 9-11; Fig. 5, top). However, such features are also present in the innermost usable apertures (apertures 16, 18, and 19; Fig. 5, middle). Although the PAH equivalent widths in these innermost apertures are small due to the bright hot dust continuum near the AGN, the fluxes of the PAH lines are several times as bright as in aperture 12 (outside the inner spiral) and typically half as bright as in the inner spiral. These innermost apertures also show the strongest [S IV $] 10.51 \mu \mathrm{m}$ emission, while [Ar II] $6.98 \mu \mathrm{m}$ and $\mathrm{H}_{2} 9.66 \mu \mathrm{m}$ emission are strongest on the inner spiral.

For comparison, the average starburst galaxy spectrum from Brandl et al. (2006) was also analyzed using PAHFIT. Line fluxes and equivalent widths measured using PAHFIT cannot be directly compared with measurements made using other techniques because PAHFIT takes into account the flux in the extended wings of the spectral lines; see Smith et al. (2007) for details. PAH equivalent widths are $2.36,7.15,1.09,1.36$, and $0.97 \mu \mathrm{m}(6.2,7.7,8.6$, 11.3 , and $12.7 \mu \mathrm{m}$ features, respectively). Although the inner 


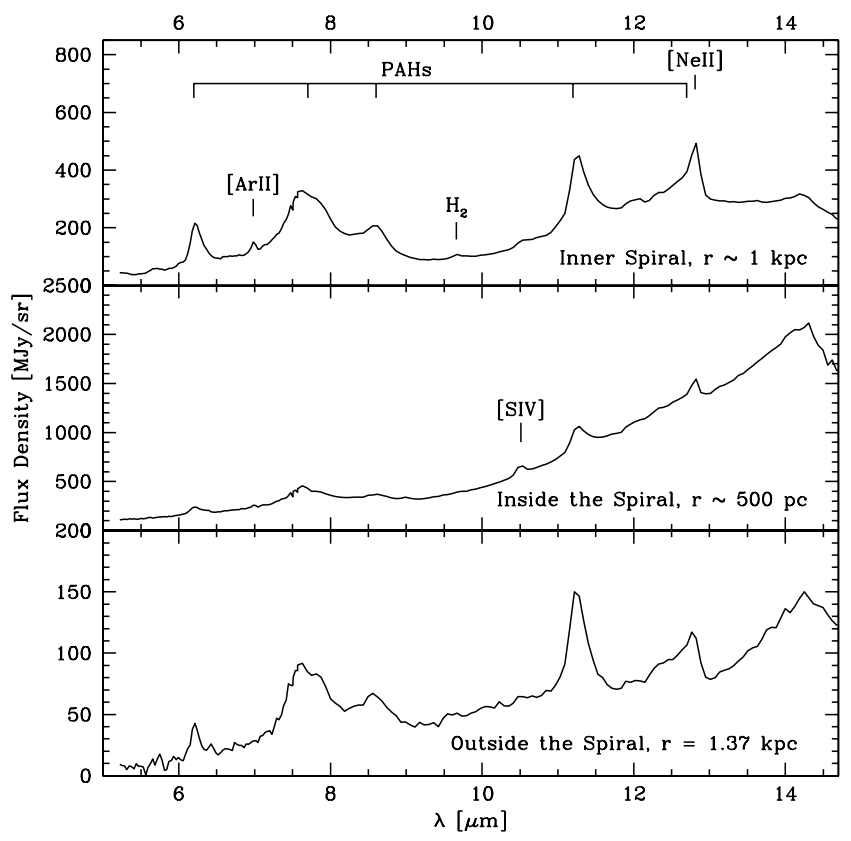

FIG. 5.- IRS SL spectra of NGC 1068. The top panel shows the average of eight apertures on the inner spiral, the middle panel shows the average of the three apertures nearest the nucleus, and the bottom panel shows aperture 12, outside the inner spiral.

spiral is the source of the strongest PAH emission in NGC 1068, this emission is significantly weaker than that seen in starburst galaxies.

With the detection of bright PAH emission lines throughout the inner spiral, the model of Bland-Hawthorn \& Voit (1993) in which the IR peaks on the inner spiral originate from dust grains heated by the AGN can be ruled out. A composite model in which the IR peaks are due to a combination of PAH emission plus continuum from hot dust heated by the AGN can also be strongly constrained. As shown in Figure 3, apertures 1 and 9 both lie within the ionization cones and are the closest apertures to the PAH knots on the inner spiral. If hot dust made a significant contribution to the flux in these apertures, the PAH equivalent widths would be lower than in apertures on regions of the inner spiral falling outside the ionization cones (apertures 3-5, 10, and 11). Instead, the PAH equivalent widths measured in apertures 1 and 9 are as high or higher than those measured elsewhere on the inner spiral. Any contribution of continuum flux from hot dust heated by the AGN must be too small to significantly affect the PAH equivalent widths. We estimate the amount of additional continuum flux within the ionization cones compared to the rest of the inner spiral by comparing the flux ratios and equivalent width ratios of the PAH features. PAH fluxes in apertures 1 and 9 are brighter than the average values in the rest of the inner spiral by factors of $20 \%-80 \%$; equivalent widths are brighter by factors of $10 \%-100 \%$. The signature of a greater contribution from the continuum is a larger increase in flux than in equivalent width. This is seen in aperture 9 , with a flux increase of $31 \%$ and equivalent width increase of $15 \%$ in the 11.3 and $12.7 \mu \mathrm{m}$ features that would be most affected by hot dust continuum. These ratios indicate a continuum increase of $1.31 / 1.15=14 \%$ compared to the average value in the rest of the inner spiral. However, in aperture 1 the increase in PAH equivalent width is greater than or equal to the increase in PAH flux for every feature, indicating a continuum level equal to or less than that seen in the rest of the inner spiral. Comparing the aperture 1 and 9 spectra to the average spectrum of the inner spiral apertures outside the ionization cones confirms the conclusion that little or

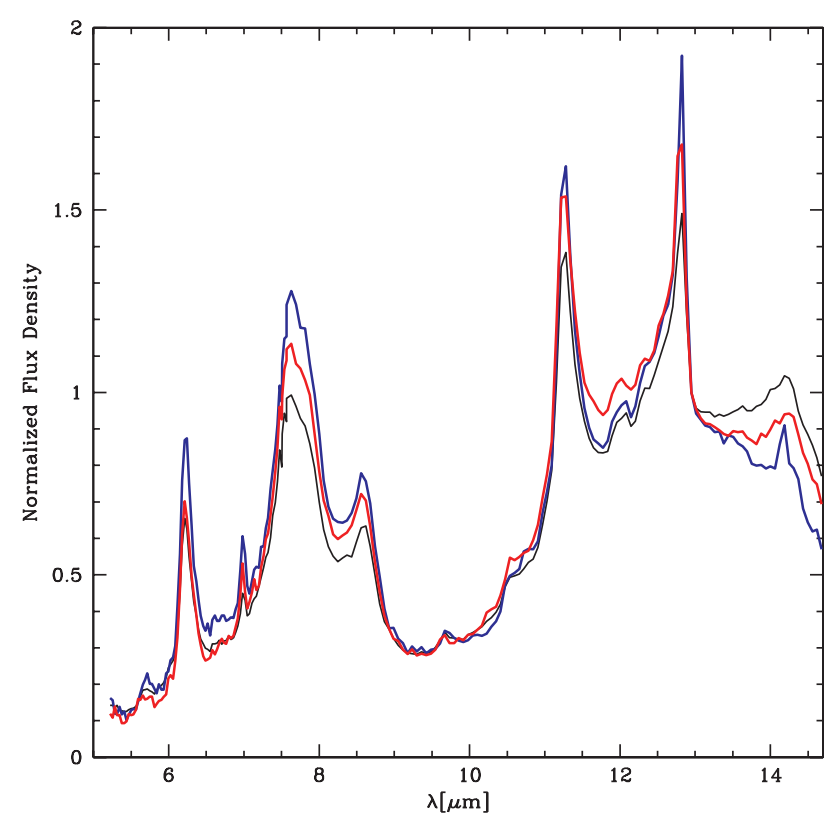

FIG. 6.-Low-resolution spectra of the inner spiral within the ionization cones (aperture 1, blue; aperture 9, red) compared with the average spectrum of the inner spiral outside the ionization cones (black). The three spectra have been normalized to a common flux density at $13 \mu \mathrm{m}$. No difference is found between the continuum flux density within the ionization cones compared to outside the ionization cones.

no excess continuum is seen within the ionization cones. Figure 6 shows the three spectra normalized to a common flux density at $13 \mu \mathrm{m}$. The apertures within the ionization cones clearly show brighter PAH features than the average spectrum, while the continuum shows very little variation between the three spectra. Warm dust visible at $24 \mu \mathrm{m}$ (Fig. 2) is present at the locations of the peaks on the inner spiral, although it is unclear whether the AGN or local star formation activity is the primary heating source.

Ratios between the 6.2, 7.7, and $11.3 \mu \mathrm{m}$ PAH features are shown in Figure 7. Lines adapted from Draine \& Li (2001) show the locations of PAH emission from a cold neutral medium (CNM) model, a warm ionized medium (WIM) model, and a photodissociation region (PDR) model. Also shown is a portion of the boundary of the region of PAH line ratios, which can be reproduced by the composite ISM model of Draine \& Li (2001). With the exception of aperture 10, the PAH measurements of the inner spiral all lie above the boundary, within the region consistent with a composite model. Aperture 5 is the outlier in the direction of a colder environment and smaller PAH molecules. The $\mathrm{H}_{2} 9.66 \mu \mathrm{m}$ line is nearly a factor of 2 brighter in this aperture than in the other apertures. Interestingly, aperture 18 - only $480 \mathrm{pc}$ from the nucleus - also lies in this cold ISM region of the diagram. It is also interesting that aperture 10 on the inner spiral and aperture 12 outside the inner spiral both lie in the region requiring more ionizing radiation than the composite ISM model, with PAH ratios similar to the majority of the apertures interior to the inner spiral. Since apertures 10 and 12 are both well outside the [O III] ionization cone, ionization from the AGN is unlikely. Aperture 10 coincides with the location of the peak $\mathrm{CO}$ emission in the map of Schinnerer et al. (2000) suggesting the formation of massive stars as a likely ionization source. The general picture presented by the ratios of PAH features is that PAH molecules in the inner spiral are smaller and lie in a cooler, less ionized environment than PAHs closer to the nucleus.

The CNM-like PAH ratios of aperture 18 suggest that radiation from the AGN may be absorbed within a few hundred parsecs 


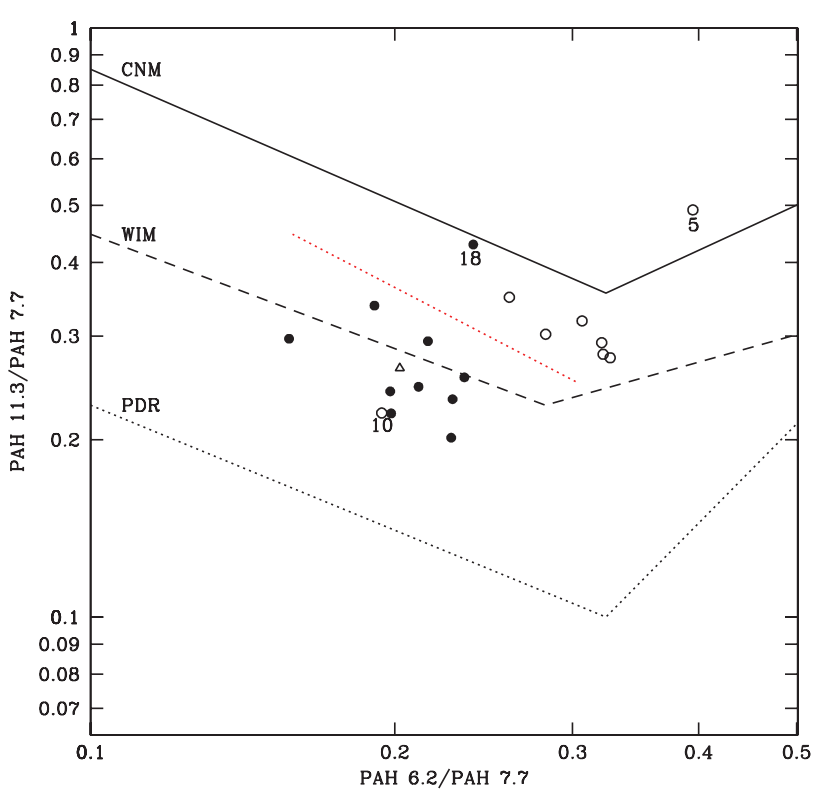

FIG. 7.- Relative strengths of three PAH emission features. Apertures along the inner spiral are shown as open circles. Apertures inside the inner spiral are shown as filled circles. The aperture exterior to the inner spiral is shown as an open triangle. Outliers have been labeled by aperture number. Uncertainties are $\sim 0.01$ in each PAH flux ratio, and are not shown. The lines are adapted from Draine \& $\mathrm{Li}$ (2001). The solid line corresponds to the CNM model, the dashed line corresponds to the WIM model, and the dotted line corresponds to the PDR model. For all three models, PAH molecule size increases to the left. The red dotted line shows part of the boundary of the region above which a simple ISM model can reproduce observed PAH features. Data below this line require greater ionization.

along the northeast ionization cone. The radio jet of Condon et al. (1990) ends abruptly near the same location. However, opticalline ratios also show that radiation from the AGN is ionizing gas up to $11 \mathrm{kpc}$ from the nucleus within the northeast cone (Veilleux et al. $2003 \S 3.2$ ).

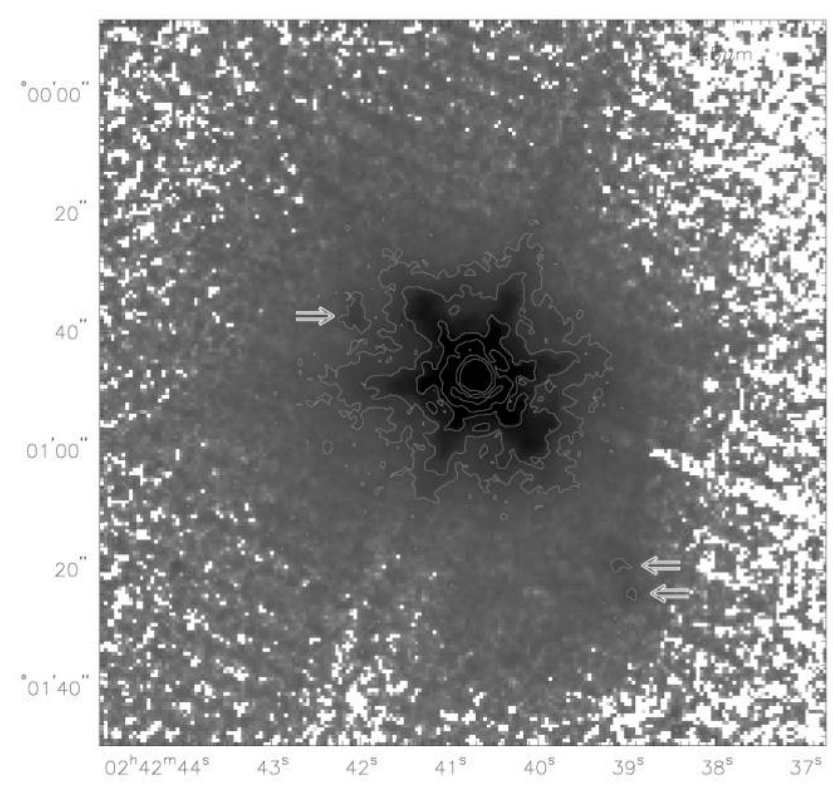

Although the $24 \mu \mathrm{m}$ MIPS data are saturated ( $\left.r<13^{\prime \prime}, 900 \mathrm{pc}\right)$, the innermost available isophotes are elongated along the same northeast-southwest axis corresponding to the arcs seen at other wavelengths (Fig. 2). In addition to the PAH knots, a weaker feature is visible in both the IRAC and MIPS $24 \mu \mathrm{m}$ images, corresponding to the northeast tip of the southern arm of the inner spiral $\left(22^{\prime \prime}\right.$ from the nucleus at P.A. $\left.=64^{\circ}\right)$. Two additional peaks are seen $\sim 45^{\prime \prime}(3 \mathrm{kpc})$ to the southwest of the nucleus at P.A. $=214^{\circ}$. The nonstellar emission map (Fig. 8) shows that hot dust is present in these locations. The subtraction of the nuclear PSF (right panel) suggests that there is little hot dust emission in the inner $\sim 20^{\prime \prime}$ outside of the nucleus; widespread heating of dust to $\sim 1000 \mathrm{~K}$ can be ruled out. It is curious that every peak of IR emission in the disk of NGC 1068, including the three such locations on the inner spiral as defined by the Schinnerer et al. (2000) CO map, lie within the ionization cones of the AGN.

\subsection{Comparison to Other Wavelengths}

The radio continuum emission (Condon et al. 1990) comes to an abrupt end at a distance of 500-700 pc from the nucleus, roughly halfway to the inner spiral, as shown in Figure 9. The $8 \mu \mathrm{m}$ knots on the inner spiral lie $\sim 5^{\prime \prime}(300 \mathrm{pc}$ ) farther along the radio axis. Cecil et al. (1990) found that the high-velocity nuclear outflow ends abruptly at the northeast radio lobe and the southwest radio hotspot ( $4.5^{\prime \prime}$ from the nucleus).

The CO observations of Schinnerer et al. (2000; Fig. 9) show a coherent spiral structure. The $8 \mu \mathrm{m}$ emission peaks coincide with regions of bright $\mathrm{CO}$ emission, although the converse is not true. The two brightest PAH knots are seen at precisely the locations where the radio jet axis intersects the inner spiral (Fig. 9). The 200-500 pc distance between the ends of the radio jets and the inner spiral indicate that the AGN is unlikely to trigger shockinduced star formation, however.

The optical emission-line imaging of Veilleux et al. (2003) provides important insight into the origin of the observed PAH knots. The PAH knots coincide with strong $\mathrm{H} \alpha$ emission (Fig. 10, left) and with small $[\mathrm{O}$ III $] / \mathrm{H} \beta$ ratios $(0.2-1.7$; Fig. 10, right $)$. The $8 \mu \mathrm{m}$

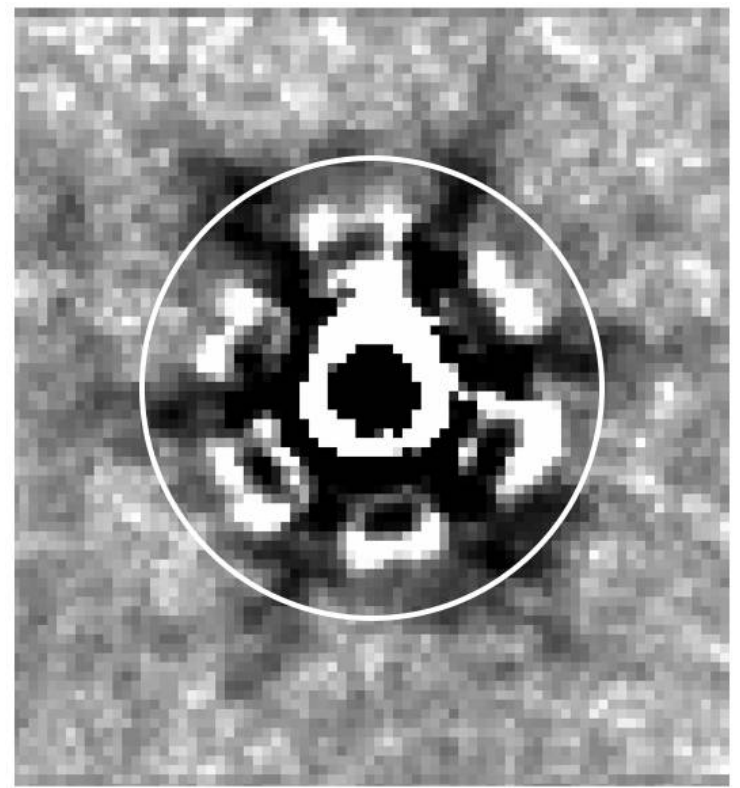

FIG. 8. - Left: Contours of an estimate of the nonstellar emission overlaid on a $4.5 \mu \mathrm{m}$ gray-scale image of NGC 1068 . As expected, the AGN dominates the nonstellar

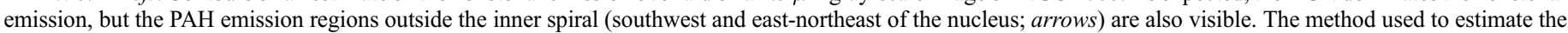

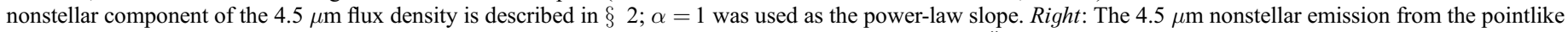

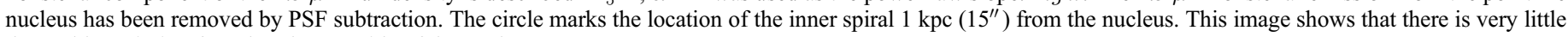
detectable emission from hot dust outside of the nucleus. 


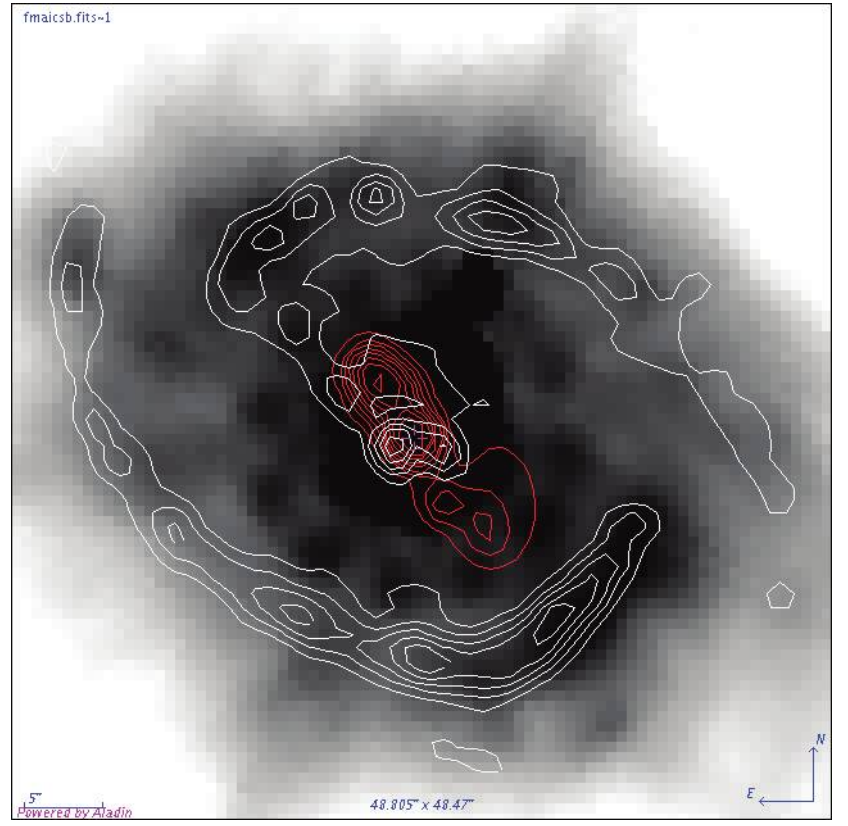

FIG. 9.-The $8 \mu \mathrm{m}$ image of the center of NGC 1068 , with $1.49 \mathrm{GHz}$ radio continuum contours from Condon et al. $(1990 ;$ red $)$ and ${ }^{12} \mathrm{CO}(1-0)$ contours from Schinnerer et al. (2000; white) overlaid. Note that the peaks of $8 \mu \mathrm{m}$ emission lie at the locations where the radio jet axis intersects the inner spiral.

and $\mathrm{H} \alpha$ images show strikingly similar morphologies, tracing active star-forming regions across a wide range in wavelength. The $[\mathrm{O}$ III $] / \mathrm{H} \beta$ ratio is an indicator of mean ionization and temperature; high ratios ( $>3$, as a rule of thumb) are typical of AGNs, while lower ratios are typical of $\mathrm{H}$ II regions (Veilleux \& Osterbrock 1987). As Veilleux et al. (2003) pointed out, high $[\mathrm{O}$ III] $/ \mathrm{H} \beta$ ratios are seen out to $R \sim 11 \mathrm{kpc}$ in NGC 1068, most visibly in the northeast ionization cone. Figure 10 clearly shows that both the starburst knots and the three fainter IR emission peaks have optical-line ratios indicative of star formation. Although the $\mathrm{H} \alpha$ and $[\mathrm{O}$ III] $/ \mathrm{H} \beta$ data confirm the conclusion of Le Floc'h et al. (2001) that the PAH knots are due to star formation in those locations on the inner spiral, it is still surprising that bright PAH emission should be present from regions that are being illuminated by ionizing radiation from the AGN.

While the galaxy disk is illuminated by the AGN, it is unclear how opaque the PAH-emitting regions are to radiation from the AGN (e.g., see Fig. 11, left). The Galaxy Evolution Explorer (GALEX) data (Gil de Paz et al. 2007) provide little guidance on this issue. Figure 11 (right) is a GALEX FUV image of NGC 1068 shown with contours of both the Spitzer $8 \mu \mathrm{m}$ and PSF-subtracted $24 \mu \mathrm{m}$ emission overlaid. As is apparent in the figure, the regions showing strong PAH and warm dust emission are clearly UV luminous, indicating that (1) the PAH-emitting regions are optically thin to UV photons and thus unshielded by the AGN, (2) the UV emanates from the optically thin outer envelope of a much more embedded, optically thick starburst responsible for producing the observed PAH emission, or (3) the UV emission is AGN light scattered by optically thick clouds. The latter possibility is unlikely; a simple assumption of a $100 \mathrm{pc}$ spherical cloud with an albedo of one would reflect a flux $\sim 10^{3}$ fainter than the flux of the AGN. Comparing measurements within 6" (GALEX FWHM) radius apertures centered on the nucleus and on each PAH knot, the GALEX data show that the PAH knots are $~ 10 \%$ as bright as the nucleus in the FUV, and therefore scattering from the AGN is unlikely to be a significant contribution. Thus, the UV emission is associated with stars, but it cannot be used to rule out whether the PAH regions are shielded from the AGN.

Further interpretation is complicated by the near-coincidence between the axis of the AGN jet and the position angle of the inner stellar bar. Intense star formation is common at the ends of a bar (Combes \& Gerin 1985), so the AGN may have no effect on the PAH knots at all. It is also possible that the AGN is either triggering increased star formation or directly exciting PAH emission. Either the high-energy photons capable of destroying PAHs are
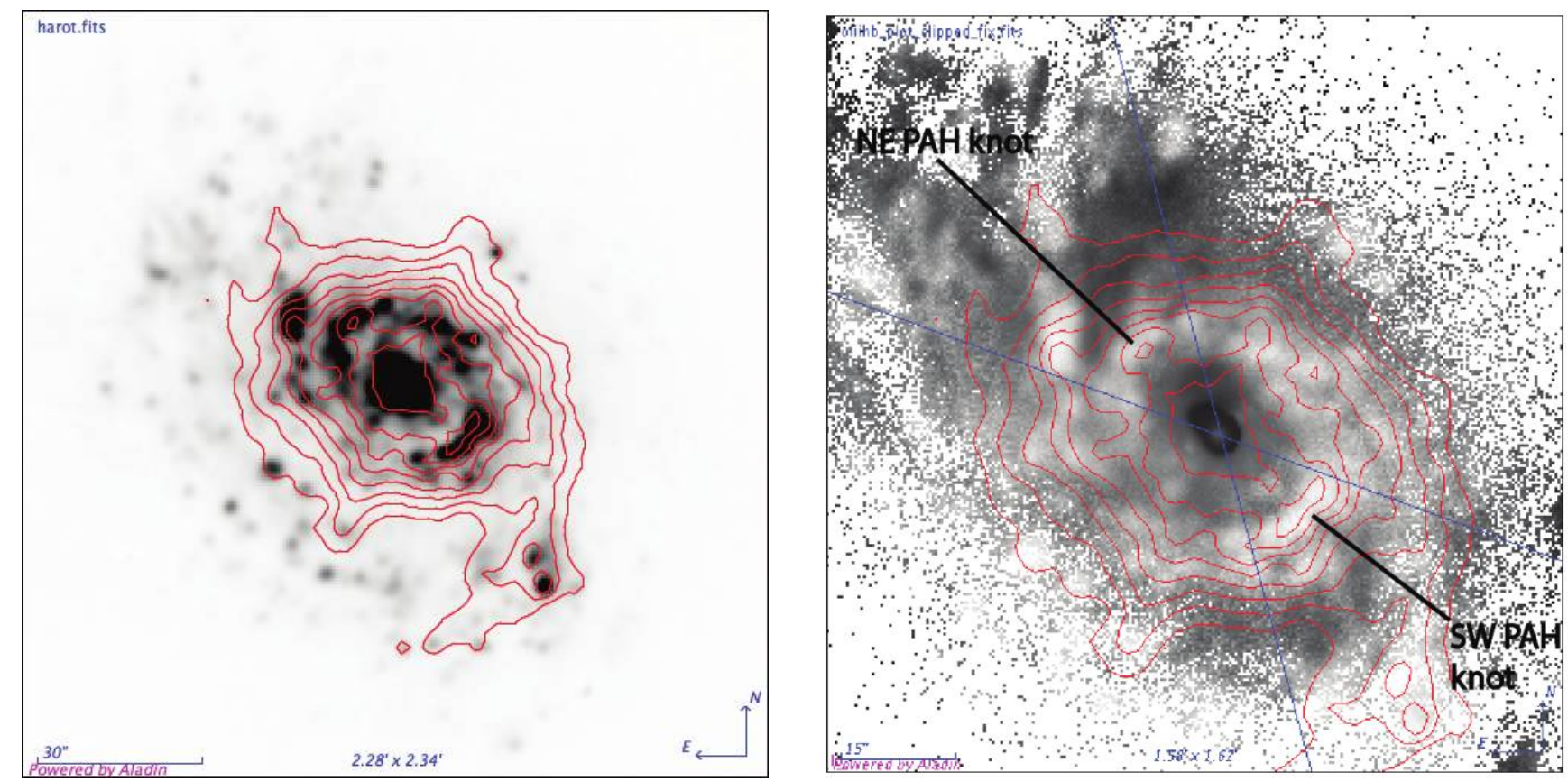

FIG. 10. - Left: $\mathrm{H} \alpha$ image of NGC 1068 from Veilleux et al. (2003) overlaid with $8 \mu \mathrm{m}$ contours. Right: [O III]/H $\beta$ image of NGC 1068 from Veilleux et al. (2003) overlaid with $8 \mu \mathrm{m}$ contours. The orientation of the ionization cones is shown, and the $\mathrm{PAH}$ knots on the inner spiral are labeled. The [O III] $/ \mathrm{H} \beta \mathrm{ratio}$ is low throughout the inner spiral (the light gray regions on the inner spiral have ratios $\sim 1$ ). Elsewhere in the ionization cone, particularly to the northeast, the $[\mathrm{O}$ III] $/ \mathrm{H} \beta \mathrm{ratio}$ is several times as large (the dark gray regions have ratios $\sim 7$ ), indicating that the AGN is the ionizing source. 

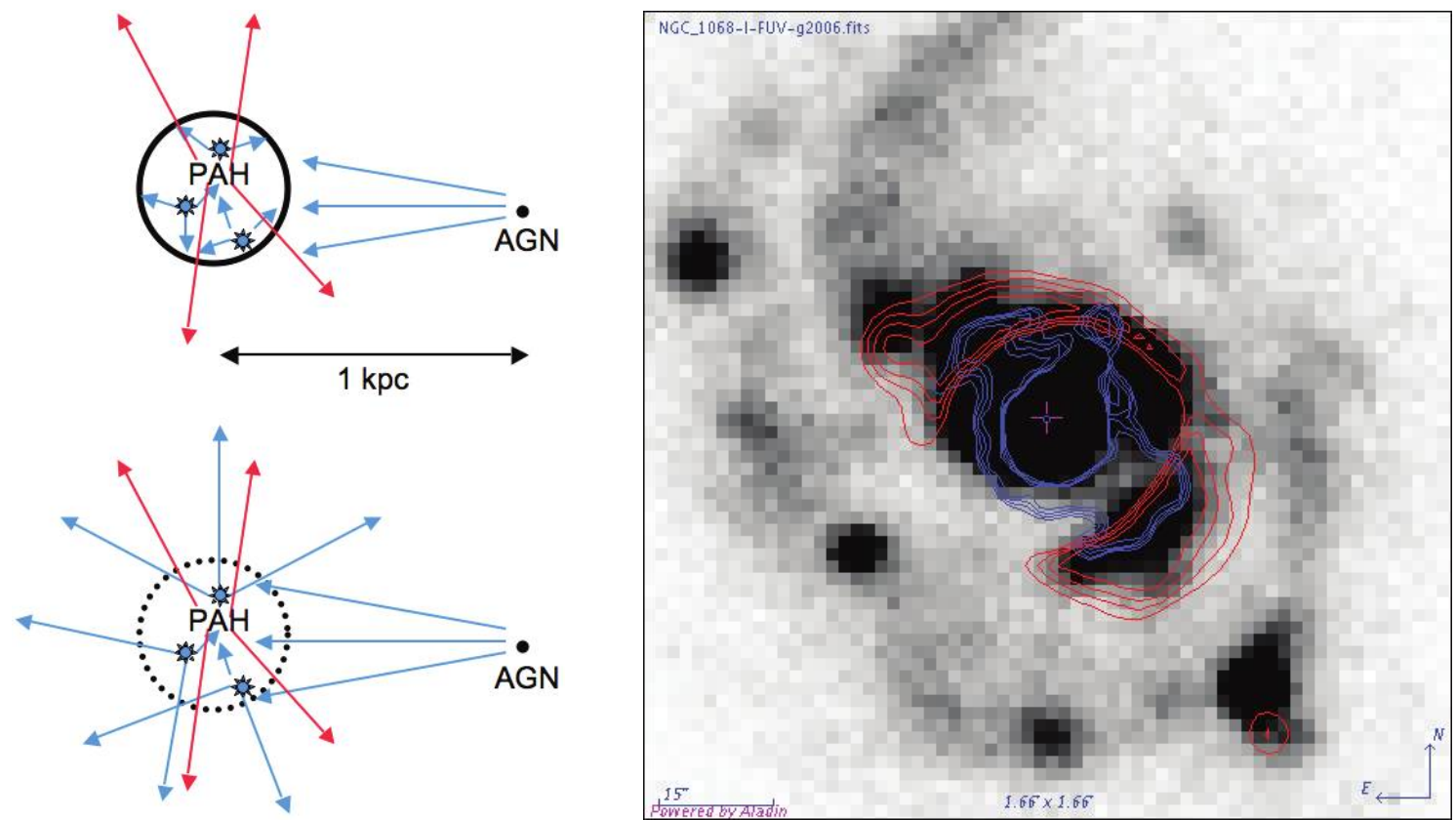

FIG. 11. - Left: Diagram showing the optically thick (top) and optically thin (bottom) scenarios described in the text. The diagram is not to scale. Blue lines represent UV photons from the AGN or massive stars; red lines represent PAH emission. Right: The GALEX FUV (1516 ̊̊; gray scale) data from Gil de Paz et al. (2007) is compared with Spitzer $8 \mu \mathrm{m}$ (blue) and PSF-subtracted $24 \mu \mathrm{m}$ (red) data. The regions of the inner spiral showing strong PAH and warm dust emission are also bright in the $\mathrm{UV}$, indicating that these star-forming regions are not optically thick to UV photons. The slight offset ( $\sim 3^{\prime \prime}$ north-northeast) between the GALEX and MIPS emission is real; the validity of the GALEX WCS solution was confirmed by plotting the positions of USNO catalog stars on the GALEX image.

absorbed near the AGN while lower energy photons are unobstructed over the entire $\sim 11 \mathrm{kpc}$ of the illuminated disk, or the high-energy photon flux within $1 \mathrm{kpc}$ produces a PAH destruction rate less than or equal to the $\mathrm{PAH}$ formation rate in the star-forming regions. For details on the parameters governing the formation and destruction of PAH molecules, see Maloney (1999). The radiativetransfer model of Siebenmorgen et al. (2004) supports the latter hypothesis, finding that even in the optically thin regime PAHs survive exposure to a $10^{11} L_{\odot}$ AGN at distances of $100 \mathrm{pc}$ or more. However, Voit (1992b) calculated that PAHs could only survive direct exposure to a $2.5 \times 10^{10} L_{\odot} \mathrm{AGN}$ at distances $\geq 14 \mathrm{kpc}$.

\section{CONCLUSIONS}

We have investigated the influence of the AGN on PAH emission in NGC 1068, a LIRG with a Seyfert 2 nucleus. Bright PAH knots are detected in the inner spiral in NGC 1068. These knots lie directly along the axis of the ionization cone, which extends well beyond the inner spiral in the disk of the galaxy. The knots also coincide with the ends of the inner stellar bar. Contrary to the model of Bland-Hawthorn \& Voit (1993) the spectra and colors of the PAH knots are consistent with starlight and PAH emission, with very little if any hot dust. Three other star-forming regions are also detected: the northeast tip of the southern arm of the inner spiral and two regions $2.5-3 \mathrm{kpc}$ to the southwest. All three regions lie within the AGN ionization cones and show PAH emission at $8 \mu \mathrm{m}$ and a slight excess of $4.5 \mu \mathrm{m}$ emission relative to star- light, suggesting dust heated by young stars. IRS spectra near the PAH knots indicate that PAHs are the dominant energy source, and show that PAH emission is present within a few hundred parsecs of the AGN. PAH line ratios on the inner spiral are generally consistent with a cool, neutral ISM, while PAH line ratios interior to the inner spiral generally indicate a more ionized medium. These data show that the AGN is not destroying all PAH molecules within the ionization cone. The enhanced PAH emission within the ionization cones indicates that the AGN is either triggering increased star formation, directly exciting the PAHs, or has no effect on the dust properties in the ionization cones.

This research has made use of the NASA/IPAC Extragalactic Database (NED), which is operated by the Jet Propulsion Laboratory, California Institute of Technology, under contract with the National Aeronautics and Space Administration. We would like to thank J. Condon for making his VLA radio continuum maps available to us, P. Shopbell for $\mathrm{H} \alpha$ and [O III] images of NGC 1068, E. Schinnerer for the CO image, B. Brandl for providing his average starburst galaxy spectrum, and J. D. Smith for assistance in using the PAHFIT software. We thank the anonymous referee for constructive comments. Support for this work was provided by NASA through contracts 1263752,1264790 , and 1267948 (D. C. K. and S. V.) issued by the Jet Propulsion Laboratory, California Institute of Technology.
Allain, T., Leach, S., \& Sedlmayr, E. 1996, A\&A, 305, 602

Antonucci, R. R. J., \& Miller, J. S. 1985, ApJ, 297, 621

Armus, L., et al. 2007, ApJ, 656, 148

Bland-Hawthorn, J., Lumsden, S. L., Voit, G. M., Cecil, G. N., \& Weisheit, J. C. 1997, Ap\&SS, 248, 177

Bland-Hawthorn, J., \& Voit, G. M. 1993, Rev. Mex. AA, 27, 73

Brandl, B. R., et al. 2006, ApJ, 653, 1129

\section{REFERENCES}

Bruhweiler, F. C., Miskey, C. L., Smith, A. M., Landsman, W., \& Malumuth, E. 2001, ApJ, 546, 866

Buchanan, C. L., Gallimore, J. F., O'Dea, C. P., Baum, S. A., Axon, D. J., Robinson, A., Elitzur, M., \& Elvis, M. 2006, AJ, 132, 401

Cecil, G., Bland, J., \& Tully, R. B. 1990, ApJ, 355, 70

Clavel, J., et al. 2000, A\&A, 357, 839

Combes, F., \& Gerin, M. 1985, A\&A, 150, 327 
Condon, J. J., Helou, G., Sanders, D. B., \& Soifer, B. T. 1990, ApJS, 73, 359

Dale, D. A., et al. 2005, ApJ, 633, 857

Draine, B. T., \& Li, A. 2001, ApJ, 551, 807

Engelbracht, C. W., Gordon, K. D., Rieke, G. H., Werner, M. W., Dale, D. A., \& Latter, W. B. 2005, ApJ, 628, L29

Fath, E. A. 1909, Popular Astron., 17, 504

Fazio, G. G., et al. 2004, ApJS, 154, 10

Freudling, W., Siebenmorgen, R., \& Haas, M. 2003, ApJ, 599, L13

Genzel, R., et al. 1998, ApJ, 498, 579

Gil de Paz, A., et al. 2007, ApJS, in press

Higdon, S. J. U., et al. 2004, PASP, 116, 975

Houck, J. R., et al. 2004, ApJS, 154, 18

Le Floc'h, E., Mirabel, I. F., Laurent, O., Charmandaris, V., Gallais, P., Sauvage, M., Vigroux, L., \& Cesarsky, C. 2001, A\&A, 367, 487

Low, F. J., \& Rieke, G. H. 1971, Nature, 233, 256

Maloney, P. R. 1999, Ap\&SS, 266, 207

Marco, O., \& Brooks, K. J. 2003, A\&A, 398, 101

Mazzarella, J., et al. 2007, AJ, submitted

Morganti, R., Killeen, N. E. B., \& Tadhunter, C. N. 1993, MNRAS, 263, 1023

Myers, S. T., \& Scoville, N. Z. 1987, ApJ, 312, L39

Neugebauer, G., Green, R. F., Matthews, K., Schmidt, M., Soifer, B. T., \& Bennett, J. 1987, ApJS, 63, 615

Packham, C., Young, S., Hough, J. H., Axon, D. J., \& Bailey, J. A. 1997, MNRAS, 288, 375

Pahre, M. A., Ashby, M. L. N., Fazio, G. G., \& Willner, S. P. 2004, ApJS, 154, 229
Rieke, G. H., \& Low, F. J. 1972a, ApJ, 176, L95 1972b, ApJ, 177, L115

Rieke, G. H., et al. 2004, ApJS, 154, 25

Roche, P. F., Aitken, D. K., Smith, C. H., \& Ward, M. J. 1991, MNRAS, 248, 606

Sanders, D. B., Mazzarella, J. M., Kim, D.-C., Surace, J. A., \& Soifer, B. T. 2003, AJ, 126, 1607

Schinnerer, E., Eckart, A., Tacconi, L. J., Genzel, R., \& Downes, D. 2000, ApJ, 533,850

Siebenmorgen, R., Krügel, E., \& Spoon, H. W. W. 2004, A\&A, 414, 123

Smith, J. D. T., et al. 2007, ApJ, 656, 770

Spinoglio, L., Malkan, M. A., Smith, H. A., González-Alfonso, E., \& Fischer, J. 2005, ApJ, 623, 123

Stern, D., et al. 2005, ApJ, 631, 163

Sturm, E., Lutz, D., Verma, A., Netzer, H., Sternberg, A., Moorwood, A. F. M., Oliva, E., \& Genzel, R. 2002, A\&A, 393, 821

Telesco, C. M., \& Decher, R. 1988, ApJ, 334, 573

Unger, S. W., Lewis, J. R., Pedlar, A., \& Axon, D. J. 1992, MNRAS, 258, 371

Usero, A., García-Burillo, S., Fuente, A., Martín-Pintado, J., \& RodríguezFernández, N. J. 2004, A\&A, 419, 897

Veilleux, S., \& Osterbrock, D. E. 1987, ApJS, 63, 295

Veilleux, S., Shopbell, P. L., Rupke, D. S., Bland-Hawthorn, J., \& Cecil, G. 2003, AJ, 126, 2185

Voit, G. M. 1992a, ApJ, 399, 495 1992b, MNRAS, 258, 841

Yuan, C., \& Kuo, C.-L. 1998, ApJ, 497, 689 